\title{
A nomogram to calculate tidal volumes for ventilation of adult patients with Acute Respiratory Distress Syndrome (ARDS)
}

\author{
Williams DJ, ${ }^{1}$ Walker JD ${ }^{2}$ \\ ${ }^{1}$ ABMU NHS Trust, Morriston Hospital, Swansea SA6 6NL, UK \\ ${ }^{2}$ Betsi Cadwaladr University Health Board, Bangor, UK
}

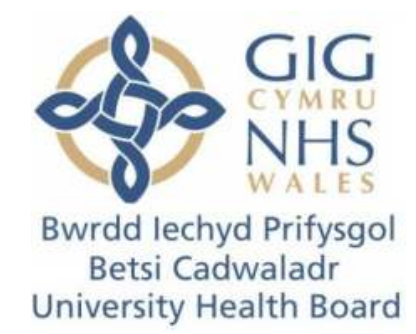

\section{Background}

High frequency low Tidal Volume (VT, mL) lung ventilation is recommended in ARDS to reduce risk of volutrauma. VT is calculated per kilogram ideal body weight (IBW, $\mathrm{kg}$ ) - typically $4 \mathrm{~mL} / \mathrm{kg}$ to 8 $\mathrm{mL} / \mathrm{kg}$ IBW - which in turn may be calculated from height $(\mathrm{cm})$ and gender.[1] This involves a multiple-stage calculation which is timeconsuming and error-prone. Smart phone apps exist to perform this calculation; [2] however smart phones are prohibited on many intensive therapy units due to infection control policies. Similar multistage calculations for drug doses can be performed more rapidly with fewer and smaller errors when a graphical calculation aid (nomogram) is used compared to an electronic calculator. [3]

We therefore developed a nomogram to perform this calculation, and validated it by comparing the graphically calculated values of VT with those produced by the gold standard of a spreadsheet (Excel, Microsoft, WA).

Fig. 1

\section{Ventilatory Tidal Volume by Height}

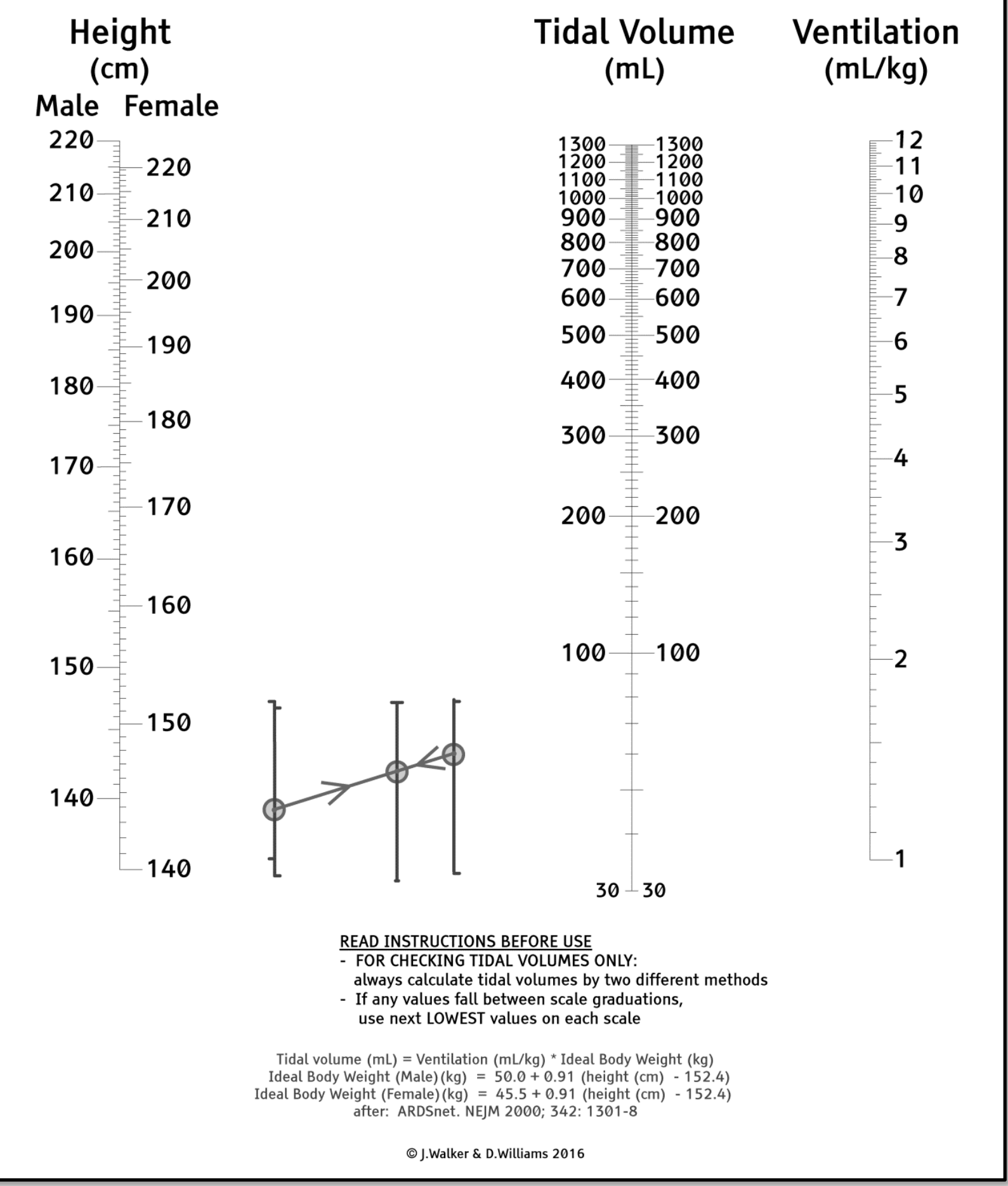

\section{Method}

The nomogram (Fig. 1) was designed using standard mathematical techniques [4] and the aid of software (www.pynomo.org). A spreadsheet was used to randomly generate 100 sets of simulated values within appropriate ranges for gender, height $(\mathrm{cm})$ and VT $(\mathrm{mL} / \mathrm{kg})$, from which the two investigators independently calculated values for VT (mL) using the nomogram. Bland-Altman (BA) analysis was used to assess accuracy, by comparing the results calculated using the nomogram with those calculated automatically by the spreadsheet; and repeatability (precision), by comparing the results obtained by the two authors, both using the nomogram.

\section{Results}

Accuracy (Fig. 2a) was: SD: 0.39\%; Bias: 0.13\%; Limits of agreement (LoA) (+/- $1.96 \mathrm{SD})$ : $-0.64 \%$ to $0.89 \%$. Repeatability (Fig. $2 \mathrm{~b}$ ) was: SD: $0.56 \%$; LoA: $-1.3 \%$ to $0.89 \%$. These were considered to be acceptable for clinical use.
Fig. 2a

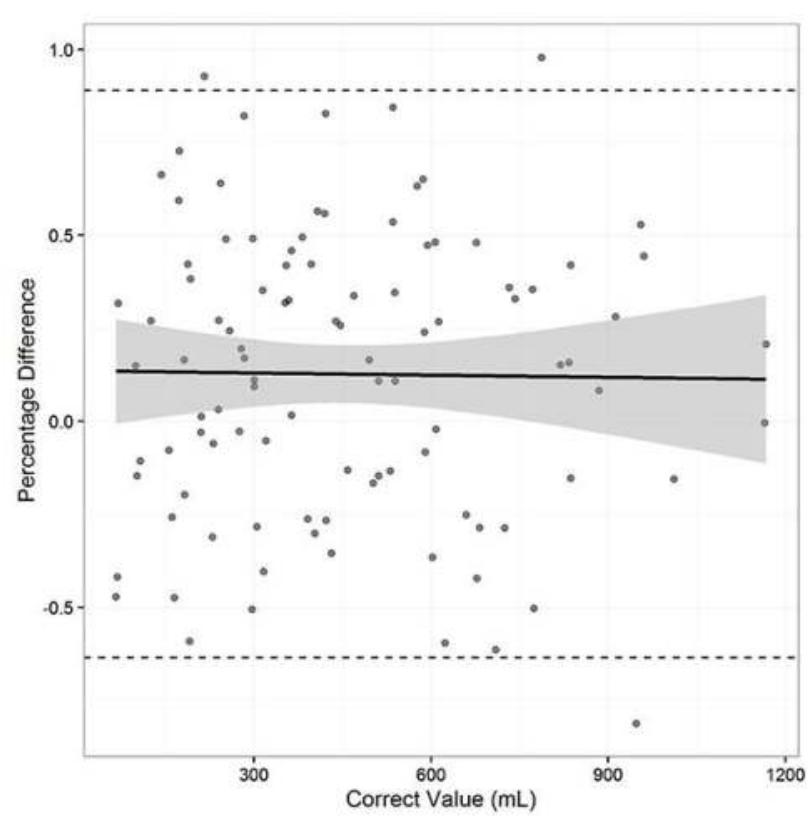

Fig. 2b

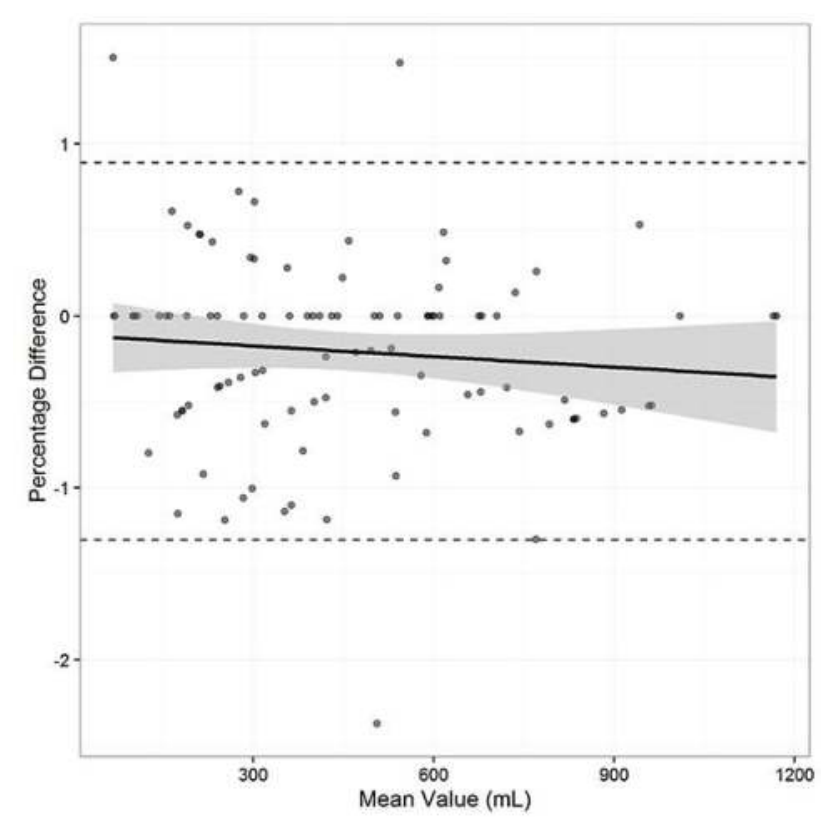

The nomogram is extremely low cost and simple to use. It may be laminated for repeated use, or individual nomograms may be printed for each patient. Lines drawn onto a printed copy simultaneously perform the calculation and provide a permanent record which may be filed in the patient notes. Nomograms are declarative - i.e. the mathematical formulae embodied in the graphical design hold for any combination of two input and one output parameters - enabling simple 'what if ?' or 'backwards' calculations to be performed.

\section{Conclusions}

The nomogram enabled VT $(\mathrm{mL})$ to be calculated from height $(\mathrm{cm})$ and gender simply and rapidly to an appropriate degree of accuracy and repeatability for clinical use.

\section{References}

[1] ARDS Network. Ventilation with Lower Tidal Volumes as Compared with Traditional Tidal Volumes for ALI and ARDS. NEJM 2000; 342: 1301-1308

[2] Owens W. The Advanced Ventilator Book. 2017. First Draught Press, USA: app available from: www.theventilatorbook.com (accessed 10 May 2017)

[3] Walker JD. The accuracy of timed maximum local anaesthetic dose calculations with an electronic calculator, nomogram, and pen and paper. Anaesthesia 2017 ;72: 760-764

[4] Allcock HJ. The Nomogram. 1950. London, Pitman. 